\title{
The utilization of intra-operative recurrent laryngeal nerve monitoring in predicting vocal fold mobility after thyroid and parathyroid surgery
}

\author{
Frank R. Miller ${ }^{*}$, Joseph D. Peterson ${ }^{1}$ and Issam Eid ${ }^{2}$ \\ ${ }^{1}$ Department of Otolaryngology-HNS, University of Texas Health Science Center San Antonio, San Antonio, TX 78229, USA \\ ${ }^{2}$ Department of Otolaryngology-HNS, University of Mississippi Medical Center, Jackson, MS, 39216, USA
}

\begin{abstract}
The role of intraoperative nerve monitoring in thyroid and parathyroid surgery has evolved over the past 15 years. Despite advances in technology there remains controversy regarding the role of nerve monitoring. The purpose of this paper was to review the relevant literature on the topic of intraoperative nerve monitoring with a particular emphasis on the efficacy of nerve monitoring to predict postoperative vocal fold mobility. In addition the paper will assess the role of nerve monitoring as an adjunct to clinical judgment regarding staging bilateral thyroid surgery when the first nerve fails to respond to intraoperative stimulation.
\end{abstract}

\section{Core Tip}

Over the last 15 years there has been controversy regarding the role of intraoperative recurrent laryngeal nerve monitoring in thyroid/ parathyroid surgery. This paper discusses the value of nerve monitoring and the role of nerve monitoring to predict postoperative recurrent laryngeal nerve function.

\section{Introduction}

Intraoperative recurrent laryngeal nerve (RLN) monitoring is a relatively new technology that has been utilized for its potential utility in helping surgeons identify the RLN, prevent and/or reduce iatrogenic nerve injury and predict post-operative vocal cord paralysis (VCP). Most authors agree that the critical factor in reducing iatrogenic injury to the RLN rests on sound anatomic knowledge and direct visualization of the nerve during surgery [1]. Over the last 10 to 15 years, the role of monitoring the RLN during endocrine surgery has been utilized in an attempt to reduce iatrogenic injury. Despite extensive use of a variety of nerve monitoring techniques, there is no clear cut evidence that routine nerve monitoring will reduce the incidence of iatrogenic nerve injury [1-11]. The utilization of IONM has also been suggested to aid in intraoperative nerve identification and provide feedback to the surgeon as they dissect and manipulate the nerve [12-33].

Another important role of intraoperative nerve monitoring is its ability to predict postoperative nerve function. This becomes particularly useful in surgical cases where intraoperative nerve dissection and traction/thermal injury may have resulted in a paresis of the nerve. In cases where the surgeon is going to dissect the contralateral nerve it is valuable to be able to reliably predict if the ipsilateral nerve is both anatomically and physiologically intact. This real time assessment of the nerve function allows the surgeon to integrate the information into intraoperative decision making, and in select cases consider staging the contralateral nerve dissection.

The purpose of this paper was to provide an overview of the role of IONM in predicting postoperative RLN function in thyroid and parathyroid surgery.

\section{Methods}

The project was initiated by performing a Pubmed search with key words (intraoperative nerve monitoring, recurrent laryngeal nerve, thyroid surgery, predicting postoperative laryngeal nerve function, etc.) focusing on the predictive nature of IONM for predicting postoperative RLN function. The authors have no competing commercial, personal, political, intellectual, or religious conflicts to declare in relation to the submitted paper. The paper is a review article and as such did not require IRB approval.

A search of the literature revealed over 300 articles related to RLN monitoring in thyroid and parathyroid surgery over the past 15 years. When the search was narrowed to IONM ability to predict postoperative RLN function there were less than 15 recent articles. A particular emphasis was placed on reviewing prospective randomized clinical trials and prospective/retrospective clinical trials. A particular emphasis was placed on analyzing studies that provided clinical results that could be utilized to guide the surgeon in intraoperative decision making (stimulus intensity and stimulus response amplitudes).

\section{Discussion}

The role of intraoperative monitoring of the RLN has been frequently debated in the literature. Whereas the efficacy of RLN in

Correspondence to: Frank R. Miller, Professor and Interim Chairman, Department of Otolaryngology-Head and Neck Surgery, University of Texas Health Science Center at San Antonio, San Antonio, TX 78229, USA, Mob: 210325-1826; Tel: 210-450-0719; Fax: 210-450-0055; E-mail: millerfr@uthscsa.edu

Key words: intraoperative nerve monitoring, nerve monitoring, thyroid surgery, parathyroid surgery, recurrent laryngeal nerve

Received: April 27, 2016; Accepted: May 25, 2016; Published: May 29, 2016 
preventing or lowering the incidence of iatrogenic nerve injury has not been shown, a number of studies point to the role of intraoperative monitoring in predicting postoperative nerve function [9-30]. Clinical studies have demonstrated that surgeons are not very accurate at predicting RLN integrity by simple observation. In several studies it has been noted that only $10 \%$ to $14 \%$ of injured nerves are identified as being injured by the operating surgeon $[22,31,32]$. Given the limitations on direct visualization to predict nerve function it is imperative to utilize new technology and objective data to assess nerve function. The key question centers on the accuracy and reliability of the intraoperative nerve monitor to predict postoperative recurrent laryngeal nerve function. In a retrospective observational study Genther et al. analyzed 997 at risk RLN [12]. They identified post dissection EMG amplitude of $200 \mathrm{uV}$ as the critical threshold to predict normal vocal cord mobility postoperatively. A stimulus response of $>200 \mathrm{uV}$ (with $1 \mathrm{mAmp}$ stimulus) had a sensitivity of $95.5 \%$ and a specificity of $99.2 \%$. The authors concluded that evoked EMG amplitudes can reliably predict immediate postoperative vocal cord function in patients undergoing thyroid and parathyroid surgery. Phelan et al. utilized continuous vagal IONM to detect signal changes in the vagal nerve/RLN complex [13]. This continuous monitoring provides real time RLN evaluation during various surgical maneuvers and can guide the surgeon to avoid specific surgical behaviors that may negatively influence nerve function. The focus of this study was to provide the surgeon with real time feedback during nerve dissection to minimize the risk of nerve paresis or injury.

Pavier et al. performed a study which assessed the ability of IONM to predict postoperative nerve function [14]. In this study of 216 RLN they identified post dissection EMG amplitude of $280 \mathrm{uV}$ (with stimulus of 1-1.5 mAmp) as predictive of normal vocal cord mobility with a sensitivity of $100 \%$ and specificity of $94.1 \%$. The risk of postoperative palsy was approximately $50 \%$ in patients with EMG amplitude less than $280 \mathrm{uV}$. Eid et al. analyzed 909 RLN at risk during thyroid and parathyroid surgery [16]. Using a post dissection stimulus of $0.5 \mathrm{mAmp}$ they demonstrated an overall sensitivity of $98.4 \%$ and a specificity of $62.5 \%$ to predict postoperative vocal cord function. No specific post dissection EMG amplitude was recorded in this study. They further identified an interesting small sub group $(n=12)$ of patients with a false positive stimulation test (positive stimulation at $0.5 \mathrm{mAmp}$ on EMG but immobile vocal cord postoperatively). Despite the false positive in this small sub group of patients eleven of the twelve recovered normal vocal cord mobility in the first 3 to 12 weeks after surgery.

In an interesting study Sadowski et al. analyzed the value of IONM in changing the operative strategy in patients undergoing planned bilateral (total) thyroidectomy [17]. In a series of 220 patients undergoing planned bilateral thyroidectomy 9 of the 220 had a loss of intraoperative EMG signals on the first surgical side. The surgeon then deferred the contralateral dissection to minimize the risk of bilateral nerve paralysis. In these 9 patients there were two false positive IONM loss. In the remaining seven all demonstrated total or partial laryngeal nerve palsy at postoperative day 1 . In all seven cases the nerve recovered over 1 to 4 months. The authors concluded that IONM can reduce the rate of bilateral laryngeal nerve palsy to near $0 \%$ if the second side is deferred to give the ipsilateral nerve time to recover. Another study by Sitges-Serra et al. took this analysis a step further in assessing the initial nerve dissected during thyroid surgery [19]. In a series of 259 patients they noted the loss of signal in 16 nerves loss of intraoperative nerve signal defined as. In 15 of the 16 nerves the signal recovered (mean amplitude of $132 \mathrm{uV}$ ) in 10-35 minutes. Only 3 of the 15 nerves that recovered its signal demonstrated transient vocal cord paresis. The loss of the intraoperative nerve signal may not be an all or none phenomenon. The authors concluded that judicious bilateral thyroidectomy may be considered even in the event that the signal is lost on the ipsilateral nerve.

Tomoda et. al, in an older study from 2006, analyzed 2197 RLN utilizing a palpation technique to assess intraoperative nerve function [20]. They stimulated the RLN with a disposable hand held nerve stimulator (setting $1.0 \mathrm{mAmp}$ ) while they performed manual palpation of the posterior cricoarytenoid muscle. This older technique demonstrated a sensitivity and specificity of $69.3 \%$ and $99.7 \%$ respectively in predicting postoperative vocal cord palsy. Even in situations where the there was no contraction of the PCA and postoperative vocal cord palsy, they noted that in the majority of cases the vocal cord will regain mobility within one year if the nerve was anatomically intact at the end of the surgical case.

Goretski et al., assessed the value of IONM in the surgical strategy in bilateral thyroid surgery [21]. They retrospectively analyzed 1333 consecutive patients with benign thyroid disease to assess the value of IONM in impacting the decision process to proceed with bilateral thyroidectomy/nerve dissection. They advocate a staged operation in the setting of an ipsilateral RLN that fails to respond to nerve stimulation at $1.0 \mathrm{mAmp}$ and an absent or reduced stimulation amplitude of less than $100 \mathrm{uV}$. This lack of response had a sensitivity and specificity of $93 \%$ and $80 \%$. This approach can minimize the risk of postoperative bilateral nerve paralysis while allowing the surgeon to complete the operation after the nerve has recovered (typically within 10-12 weeks).

Caragacianu et al., performed a very interesting study to determine normative nerve monitoring values associated with normal postoperative glottis function [22]. In a series of 125 patients with 167 nerves at risk they identified a stimulus amplitude of $247 \mathrm{uV}$ (with a stimulus level of $1.0 \mathrm{mAmp}$ ) as the threshold that can positively predict normal vocal cord mobility. In cases which the nerve stimulus response amplitude is less than $247 \mathrm{uV}$ (and clearly if $<100 \mathrm{uV}$ ) the surgeon can consider delaying the surgery on the contralateral side to lower the risk of bilateral RLN injury and subsequent bilateral vocal cord paralysis.

In a recent study Faden et al. analyzed the optimal stimulation threshold to predict postoperative vocal cord mobility [30]. The stimulus threshold was set at $150 \mathrm{mV}$ and incremental stimulation levels were utilized at $0.3 \mathrm{mAmp}, 0.5 \mathrm{mAmp}, 0.8 \mathrm{mAmp}$, and 1.0 $\mathrm{mAmp}$. A stimulation threshold of $0.5 \mathrm{mAmp}$ optimized the predictive value of nerve monitoring (sensitivity $68 \%$ and specificity $84 \%$ with excellent positive predictive value) but stimulation at multiple levels significantly improves the overall predictive value of IONM. The lower level of stimulation at $0.3 \mathrm{mAmp}$ had high sensitivity but low specificity and low positive predictive value meaning that low levels of stimulation are excellent at confirming that the RLN that stimulates at $0.3 \mathrm{mAmp}$ will have normal function postoperatively. As the stimulus intensity increases the sensitivity decreases and the specificity rises. This paper also discusses some of the complex issues involved in assessing the response of the nerve to stimulation including different IONM techniques, accurate assessment of RLN function postoperatively, location of stimulus application to RLN, and the role of various stimulation thresholds on the predictive value of IONM.

As with any technology there are limitations with IONM of the RLN. Nerve monitoring requires accurate placement of an endotracheal tube with surface electrodes in contact with the vocal folds. The accuracy of IONM depends on accurate utilization of the system and an understanding how to trouble shoot problems that may arise. This 
technology requires the surgeon to obtain the necessary training and experience to use the system efficiently and accurately.

Some authors have suggested selective use of the IONM system for the "difficult cases" but our opinion is that if a surgeon plans on using IONM then one should strongly consider using it in all cases. Even small tumors may be adherent to RLN and it is difficult to predict the difficult case prior to surgery. The routine utilization of IONM allows the surgeon to steepen the learning curve and improve interpretive skills and ability to troubleshoot system malfunction [35]. The time to set up the system becomes minimized with routine use and in most cases requires less than 5 to $10 \mathrm{~min}$ to set up and troubleshoot. The cost of IONM is minimal and mainly related to the cost of the nerve monitoring endotracheal tube (typical cost approximately $\$ 150$ in US dollars).

\section{Conclusion}

1. The risk of RLN injury during thyroid/parathyroid surgery ranges from $2 \%$ to $4 \%$ in experienced surgical hands.

2. There is no evidence that routine IONM of the RLN can reduce the risk of iatrogenic RLN injury.

3. IONM is a valuable adjunct to RLN identification but does not replace an understanding of the detailed surgical anatomy.

4. IONM can be a valuable tool to help the surgeon predict postoperative vocal fold mobility and postoperative paresis/paralysis.

5. The optimal nerve stimulus intensity to assess the RLN is 0.5 mAmp to $1.0 \mathrm{mAmp}$. A nerve stimulus response greater than $200 \mathrm{uV}$ can reliably predict normal vocal fold mobility postoperatively with an excellent positive predictive value of $95 \%$ to $99 \%$.

6. The information obtained from IONM can guide the surgeon to proceed with bilateral thyroidectomy (bilateral RLN dissection) or to consider staged surgery when the nerve stimulus response is less than $150 \mathrm{uV}$ to $200 \mathrm{uV}$.

7. Further research should evaluate methods of incorporating standardized stimulation and response amplitude values for IONM as intermittent fluctuations throughout surgery may have a predictive value.

\section{References}

1. Steurer M, Passler C, Denk DM, Schneider B, Niederle B, Bigenzahn W (2002) Advantages of recurrent laryngeal nerve identification in thyroidectomy and parathyroidectomy and the importance of preoperative and postoperative laryngoscopic examination in more than 1000 nerves at risk. Laryngoscope 112: 124-133. [Crossref]

2. Chan W, Lo CY (2006) Pitfalls of intraoperative neuromonitoring for predicting postoperative recurrent laryngeal nerve function during thyroidectomy. World J Surg 30: 806-812. [Crossref]

3. White WM, Randolph GW, Hartnick CJ, Cunningham MJ (2009) Recurrent laryngeal nerve monitoring during thyroidectomy and related cervical procedures in the pediatric population. Arch Otolaryngol Head Neck Surg 135: 88-94. [Crossref]

4. Robertson ML, Steward DL, Gluckman JL, Welge J (2004) Continuous laryngeal nerve integrity monitoring during thyroidectomy: does it reduce risk of injury? Otolaryngol Head Neck Surg 131: 596-600. [Crossref]

5. Chan W, Lang BH, Lo C (2006) The role of intraoperative neuromonitoring of recurrent laryngeal nerve during thyroidectomy: A comparative study on 1000 nerves at risk. Surgery 140: 866-873. [Crossref]

6. Cavicchi O, Caliceti U, Fernandez IJ, Macri G, DiLieto C, et al. (2009) the value of neurostimulation and intraoperative nerve monitoring of the inferior laryngeal nerve in thyroid surgery. Otolaryngol Head Neck Surg 140: 866-870. [Crossref]

7. Shindo M, Chheda NN (2007) Incidence of vocal cord paralysis with and without recurrent laryngeal nerve monitoring during thyroidectomy. Arch Otolaryngol Head Neck Surg 133: 481-485. [Crossref]

8. Alon EE, Hinni ML (2009) Transcricothyroid electromyographic monitoring of the recurrent laryngeal nerve. Laryngoscope 119: 1918-1921. [Crossref]

9. Otto RA, Cochran CS (2002) Sensitivity and specificity of intraoperative laryngeal nerve simulation in predicting postoperative nerve paralysis. Ann Otol Rhinol Laryngol 111: 1005-1007. [Crossref]

10. Hermann M, Hellabart C, Freissmuth M (2004) Neuromonitoring in thyroid surgery; Prospective evaluation of intraoperative electrophysiological responses for the prediction of recurrent laryngeal nerve injury. Ann Surg 240: 9-17. [Crossref]

11. Eid I, Miller FR, Rowan S, Otto RA (2013) The role of nerve monitoring to predict postoperative recurrent laryngeal nerve function in thyroid and parathyroid surgery. Laryngoscope 123: 2583-2586. [Crossref]

12. Genther DJ, Kandil EH, Noureldine SI, Tufano RP (2014) Correlation of final evoked potential amplitudes on intraoperative electromyography of the recurrent laryngeal nerve with immediate postoperative vocal fold function after thyroid and parathyroid surgery. JAMA Otolaryngol Head and Neck Surg 140: 124-128. [Crossref]

13. Phelan E, Schneider R, Lorenz K, Dralle H, Kamani D, Potenza A, et al. (2014) Continuous vagal IONM prevents recurrent laryngeal nerve paralysis by revealing initial EMG changes of impending neuropraxic injury: A prospective, multicenter study. Laryngoscope 124: 1498-1505. [Crossref]

14. Pavier Y, Saroul N, Pereira B, Tauveron I, Gilain L, Mom T (2014) Acute prediction of laryngeal outcome during thyroid surgery by electromyographic laryngeal monitoring. Head Neck 37: 835-839. [Crossref]

15. Thomusch O, Sekulla C, Machens A, Neumann HJ, Timmermann W, et al. (2004) Validity of intra-operative neuromonitoring signals in thyroid surgery. Langenbecks Arch Surg 389: 499-503. [Crossref]

16. Randolph GW, Dralle H; International Intraoperative Monitoring Study Group, Abdullah H, Barczynski M, Bellantone R, Brauckhoff M, et al. (2011) Electrophysiologic recurrent laryngeal nerve monitoring during thyroid and parathyroid surgery: international standards guideline statement. Layngoscope 121: S1-S16. [Crossref]

17. Sadowski SM, Soardo P, Leuchter I, Robert JH, Triponez F, et al. (2013) Systematic use of recurrent laryngeal nerve neuromonitoring changes the operative strategy in planned bilateral thyroidectomy. Thyroid 23:329-333. [Crossref]

18. Lorenz K, Sekulla C, Schelle J, Schmeiss B, Brauckhoff M, Dralle H, et al. (2010) What are normal quantitative parameters of intraoperative neuromonitoring (IONM) in thyroid surgery? Langenbecks Arch Surg 395: 901-909. [Crossref]

19. Sitges-Serra A, Fontane J, Duenas JP, Dugue CS, Lorente L, Trillo L, et al. (2013) Prospective study on loss of signal on the first side during neuromonitoring of the recurrent laryngeal nerve in total thyroidectomy. Br J Surg 100: 662-666. [Crossref]

20. Tomoda C, Hirokawa Y, Uruno T, Takamura Y, Ito Y, Miya A, (2006) Sensitivity and specificity of intraoperative recurrent laryngeal nerve stimulation test for predicting vocal cord palsy after thyroid surgery. World J Surg 28: 755-760. [Crossref]

21. Goretzki PE, Schwarz K, Brinkmann J, Wirowski D, Lammers BJ (2010) The impact of intraoperative neuromonitoring (IONM) on surgical strategy in bilateral thyroid disease: is it worth the effort? World J Surg 34: 1274-1284. [Crossref]

22. Caragacianu D, Kamani D, Randolph GW (2013) Intraoperative monitoring: normative range associated with normal postoperative glottis function. Laryngoscope 123: 30263031. [Crossref]

23. Cernea CR, Brandão LG, Hojaij FC, De Carlucci D Jr, Brandão J, et al. (2012) Negative and positive predictive values of nerve monitoring in thyroidectomy. Head Neck 34: 175-179. [Crossref]

24. Julien N, Mosnier I, Bozorg Grayeli A, Nys P, Ferrary E, Sterkers O (2012) Intraoperative laryngeal nerve monitoring during thyroidectomy and parathyroidectomy: A prospective study. Eur Ann Otorhinolaryngol Head Neck Dis 129: 69-76. [Crossref]

25. Potenza AS, Phelan EA, Cernea CR, Slough CM, Kamani DV, Darr A, et al. (2013) Normative intra-operative electrophysiologic waveform analysis of superior laryngeal nerve external branch and recurrent laryngeal nerve in patients undergoing thyroid surgery. World J Surg 37: 2336-2342. [Crossref]

26. Pisanu A, Porceddu G, Podda M, Cois A, Uccheddu A (2014) Systematic review with meta-analysis of studies comparing intraoperative neuromonitoring of recurrent laryngeal nerves verses visualization alone during thyroidectomy. J Surg Res 188: 152 161. [Crossref] 
Miller FR (2016) The utilization of intra-operative recurrent laryngeal nerve monitoring in predicting vocal fold mobility after thyroid and parathyroid surgery

27. Angelos P (2009) Recurrent laryngeal nerve monitoring: state of the art, ethical and legal issues. Surg Clin North Am 89: 1157-1169. [Crossref]

28. Calo PG, Medas F, Tatti A, Pittau MR, Demontis R, Favoriti, Nicolosi A (2013) Intraoperative recurrent laryngeal nerve monitoring in thyroid surgery: is it really useful? Clin Ter 3: 193-198. [Crossref]

29. Smith J, Douglas J, Smith B, Dougherty T, Ayshford C (2014) Assessment of recurrent laryngeal nerve function during thyroid surgery. Ann R Coll Surg Engl 96: 130-135. [Crossref]

30. Faden DL, Orloff LA, Ayeni T, Fink DS, Yung K (2014) Stimulation threshold greatly affects the predictive value of intraoperative nerve monitoring. Laryngoscope 125: 1265-1270. [Crossref]

31. Patow CA, Norton JA, Brennan MF (1986) Vocal cord paralysis and reoperative parathyroidectomy. A prospective study. Ann Surg 203: 282-285. [Crossref]

32. Lo CY, Kwok KF, Yuen PW (2000) A prospective evaluation of recurrent laryngeal nerve paralysis during thyroidectomy. Arch Surg 135: 204-207. [Crossref]

33. Randolph GW (2013) Surgical anatomy and monitoring of the recurrent laryngeal nerve. In: Randolph GW: Surgery of the thyroid and parathyroid glands. Philadelphia: Elsevier Saunders 333-334.

Copyright: $(02016$ Miller FR. This is an open-access article distributed under the terms of the Creative Commons Attribution License, which permits unrestricted use, distribution, and reproduction in any medium, provided the original author and source are credited. 